\title{
RadFET dose response in the CHARM mixed-field: FLUKA MC simulations
}

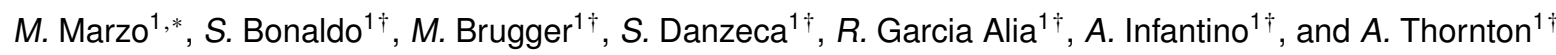 \\ ${ }^{1}$ CERN, European Organization for Nuclear Research, Geneva, Switzerland.
}

\begin{abstract}
This paper focuses on Monte Carlo simulations aiming at calculating the dose response of the RadFET dosimeter, when exposed to the complex CHARM mixed-fields, at CERN. We study how the dose deposited in the Gate Oxide $\left(\mathrm{SiO}_{2}\right)$ of the RadFET is affected by the energy threshold variation in the Monte Carlo simulations as well as the materials and sizes of scoring volumes. Also the characteristics of the input spectra will be taken into account and their impact on the final simulated dose will be studied. Dose variation as a function of the position of the RadFET in the test facility will be then examined and comparisons with experimental results will be shown. The contribution to the total dose due to every single particles of the mixed-field, under different target-shielding configurations, will be finally presented, aiming at a complete characterization of the RadFETs dose response in the CHARM mixed-fields.
\end{abstract}

\section{Introduction}

We present Monte Carlo calculations of the dose response of the RADiation sensing Field Effect Transistors (RadFET) [3] [4] [6] [9], when they are exposed to the radiation environment at the CERN High Energy Accelerator Mixed-field (CHARM) test facility [8].

The main purpose of the facility is to replicate different radiation environments (space, atmospheric, accelerator complexes, for instance) for radiation effects testing on electronic components and systems. RadFETs at CHARM are then used as online dosimeters during the radiation tests, to characterize the complex mixed-fields reproduced in the test area.

In this regard, we are interested in investigating the dose deposited in the Gate Oxide (GO) of the RadFET, a very small sensitive volume where the increase of the accumulated charge $\delta Q_{t o t}$, as a consequence of the exposure to the radiation fields of interest, implies a change of the sourcedrain channel thickness of the MOSFET. This causes a drift in the threshold voltage $\delta V_{t h}$ of the device and a modification of its electric response, which can therefore be correlated to the deposited dose.

Measurements and FLUKA [1] [2] simulations of the dose response of RadFETs in the mixed-field at CHARM had been already performed to understand the radiation field in the test area, but discrepancies in the $25-45 \%$ range had been identified between simulated and experimental values. In this paper we want to set up more accurate simulations to investigate this mismatch.

\footnotetext{
Author's e-mail: matteo.marzo@cern.ch

$\dagger$ Please contact the author, if you need the coauthors' mail addresses
}

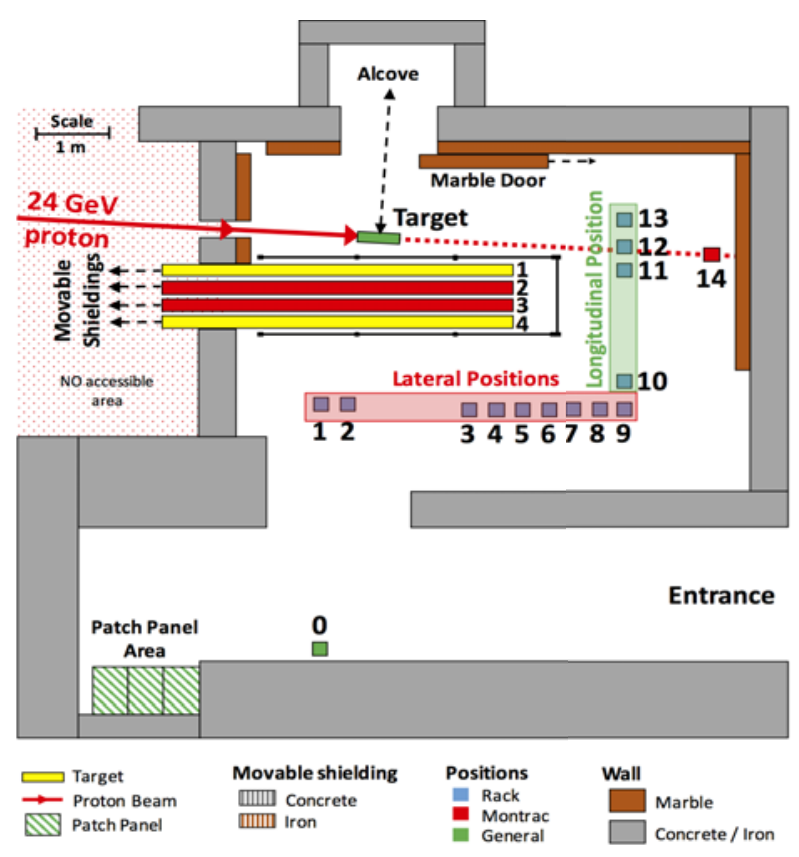

Figure 1: CHARM test facility model, top view.

\section{CHARM and its mixed-field: measurements VS FLUKA simulations}

The CHARM test facility [8] is located in the Meyrin site of CERN, specifically in the Proton Synchrotron (PS) East Area hall. The main source of the radiation field reproduced at CHARM is in fact the shower developing from the interaction between the $24 \mathrm{GeV} / \mathrm{c}$ protons beam extracted from the PS and a metallic target. 


\subsection{Facility variables}

Due to the high energetic particles treated at CHARM, particular attention has been given to the protection of the personnel, by using concrete, marble and iron as materials surrounding the central part of the facility itself and separating the irradiation chamber, from the technical area [8] [10]. The irradiation chamber is a $7 \times 7 \times 3 \mathrm{~m}^{3}$ room, available for the irradiation tests.

The 4 most relevant variables at CHARM, needed to obtain and properly tune the mixed-field of the facility, are:

- The $24 \mathrm{GeV} / \mathrm{c}$ beam from the PS;

- A target- Copper (cp), Aluminum (al) or Aluminum with holes (alh), depending on the wanted mixed-field intensity and composition, as it will be clear in the following- placed along the beam direction (Fig. 1);

- 13 rack test locations in lateral and downstream positions with respect to the beam direction, averagely $3 \div 5$ $\mathrm{m}$ distant from the target (Fig. 1);

- A shielding between the target and the lateral positions created by using 4 movable blocks of concrete (C) and iron (I) (Fig. 1).

Entering the CHARM facility, in fact, the the $24 \mathrm{GeV} / \mathrm{c}$ protons beam impinges against the above mentioned target. From the interaction between the two, a shower of particles takes place. This shower can further interact with the shielding (if any), possibly generating other secondaries and consequently producing (or stopping) other particles: this is the way we create and tune the mixed-field at CHARM.

Changing the target material, the shielding configuration and having the possibility of testing the electronics at many test locations, different radiation fields in terms of composition and intensity can be reproduced. In particular, in this paper, two target-shielding layouts will be examined: copper target and no shielding (movable plates in OFF position), namely cp_0000, and copper target and concrete-iron-iron-concrete shielding, i.e. cp_CIIC. Other mixed-fields can be originated using different shielding configurations and different target materials.

FLUKA Monte Carlo simulations have already been performed to characterize the different radiation fields: the knowledge of the field at any rack test locations in terms of particles spectra (coming from the previous simulations) is the first, preliminary step to run the dedicated simulations on the RadFET.

\subsection{FLUKA simulations settings to calculate the mixed-field at CHARM}

A FLUKA model of CHARM was built to simulate the radiation field inside the test facility and retrieve all the most relevant quantities for radiation to electronics testing. The NEWDEFA default in FLUKA (characterized by particles transport thresholds at $10 \mathrm{MeV}$, except for neutrons at $10^{-5} \mathrm{eV}$ and delta ray production with threshold at $1 \mathrm{MeV}$ ) had been used as a first approximation to get reasonable results, saving CPU time, given the size of the geometry. The use of NEWDEFA was crucial to reduce the computational time, given the large geometry of the CHARM test facility (the higher the thresholds for production and transport of particles, the lower the computational time).

For the same reasons, an air cube of $20 \mathrm{~cm}$ side had been chosen as sensitive volume: scoring, for instance, dose inside a volume having the same size as the actual size of the Gate Oxide (GO) of the RadFET -whose characteristic dimension is several order of magnitude lower than the CHARM's size-, would have led to a disproportional increase of the CPU time (a larger amount of primaries to get the same statistics).

An example of the characterization of the mixed-field at CHARM in terms of simulated particle energy spectra (lethargy), for the cp_0000 and cp_CIIC configurations is presented- for the position 1- in Fig. 2. It is clearly visible how the field is populated by secondary electrons, positrons, muons $(\mu \pm)$, photons, neutrons and charged hadrons (protons, kaons $(\kappa \pm)$ and pions $(\pi \pm)$ ). Those spectra have been obtained from FLUKA, making the 24 $\mathrm{GeV} / \mathrm{c}$ proton beam interact with a copper target and simulating the entire CHARM geometry, reproducing the two different shielding layouts.
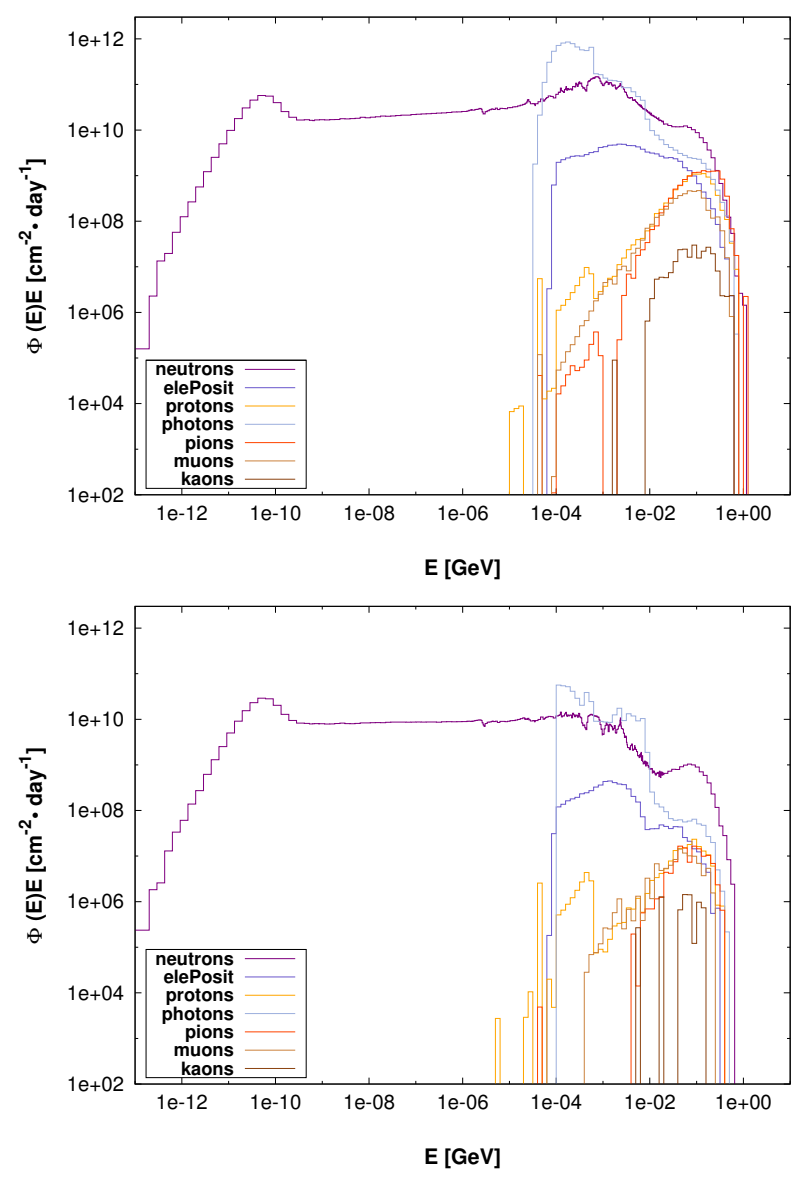

Figure 2: Mixed-field: simulated spectra (lethargy), position 1, cp_0000 and cp_CIIC configurations. 
It is clear for instance how, from Fig. 2, in the shielded configuration the lethargy $\left(\mathrm{cm}^{-2} \cdot \mathrm{day}^{-1}\right.$, considering that nominal daily value of protons on target- pot- is $1.15 \cdot 10^{15}$, during the runs) is overall lower for all particles of the mixed-field, if compared to the cp_0000 configuration: the CIIC walls are stopping a certain amount of particles of the shower, preventing them from reaching the rack position 1 , even if they are generating other secondaries.

\subsection{Experimental measurements and simulated dose: benchmark}

Dose at CHARM is experimentally detected by an integrated dosimetry system, called RadMon (Radiation Monitoring system) [5] [7]. The RadMon executes an electronic readout of the threshold voltage of the RadFETs and sends the voltage value to the CERN database. The conversion from voltage $\left(V_{g s}\right)$ to dose $(G y)$ is post-processed by means of a calibration curve, obtained for each RadFET batch in a Co60 source. The experimental results presented in this paragraph refer to the data measured during 2015 run period of CHARM, for the copper target and no shielding configuration, measured by $100 \mathrm{~nm}$ p-channel RadFET on RadMon in different test positions. The experimental doses in Tab. 1 are averages of the calibration factors retrieved during different runs of 2015 plus minus total associated errors, which take into account primary proton beam intensity error, RadFET error and standard deviation with respect to the average value [10].

It's worth noticing that the previous FLUKA simulations results (obtained by scoring the dose in a cubic volume of air, $20 \mathrm{~cm}$ side, placed in the different test positions, considering the beam-target interaction and the whole CHARM geometry, and setting energy thresholds for transport and production of secondaries at $1 \mathrm{MeV}$ ) in Tab. 1 are systematically overestimating the measured doses of $25 \%$ and $43 \%$.

\begin{tabular}{|l|lll|}
\hline Position & Exp. dose & $1^{\text {st }}$ step dose & \% diff. \\
\hline $\mathbf{1}$ & $0.776 \pm 23.0 \%$ & $1.101 \pm 0.8 \%$ & $30 \%$ \\
$\mathbf{3}$ & $1.510 \pm 23.0 \%$ & $2.281 \pm 0.6 \%$ & $34 \%$ \\
$\mathbf{5}$ & $1.190 \pm 22.0 \%$ & $2.110 \pm 0.6 \%$ & $43 \%$ \\
$\mathbf{7}$ & $1.460 \pm 23.0 \%$ & $2.260 \pm 0.5 \%$ & $35 \%$ \\
$\mathbf{1 0}$ & $1.550 \pm 29.0 \%$ & $2.401 \pm 0.5 \%$ & $35 \%$ \\
$\mathbf{1 3}$ & $3.420 \pm 28.0 \%$ & $4.510 \pm 0.3 \%$ & $24 \%$ \\
\hline
\end{tabular}

Table 1: Experimental and $1^{\text {st }}$ step simulated doses in $10^{-14} \mathrm{~Gy} / \mathrm{pot}$, in different positions at CHARM, cp_0000 configuration.

Fig. 3 shows a plot of the trend of dose simulated in different positions in the test area. The errors associated to the simulated values (as also clear from Tab. 1) are low if compared to the experimental ones, since they take into account only statistical uncertainties and no systematic errors, like in the case of measured doses.

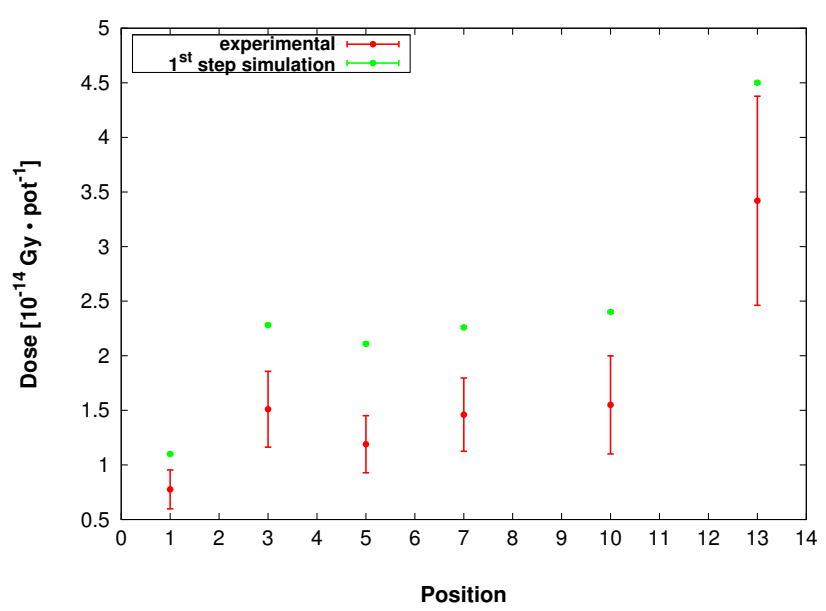

Figure 3: Experimental and simulated doses at different test positions, cp_0000 target-shielding layout.

\section{The RadFET model and the second step simulations: benchmarks}

Starting from the mismatch between the performed FLUKA simulations and the measurements detected by the RadMon, focusing on position 1 and cp_0000 layout, we want now to investigate these discrepancies and evaluate if it's possible to better simulate the radiation dose levels at CHARM. If we can improve simulations settings, aiming at obtaining doses that are more realistic and in line with experimental measurements, we could use our calculations to predict the radiation field at each position and for each target-shielding layout at CHARM, even out of the 14 ordinary predefined positions.

In order to improve the reliability of our MC simulations and the agreement with respect to the experimental data, we decide to implement a more realistic second step simulation acting on:

- Geometry and materials of the sensitive volume;

- Energy thresholds for production and transport of particles;

- Energy cutoffs for input spectra.

We want to conduct different parametric analyses to find out which are the most accurate FLUKA simulation setting, aiming at simulating the impact of the CHARM mixed-field on the RadFET oxide, through a two step simulation.

In the following paragraph, the first step dose calculated in correspondence of the position 1, for the copper target and no shielding configuration (Sect. 2.2), is taken as reference and its value is $1.101 \cdot 10^{-14} \pm 0.8 \%$ Gy/pot (protons on target, meaning each proton from the PS beam and interacting with the target- $1.15 \cdot 10^{15}$ pot/day).

Before going through the details of the parametric simulations that aim at finding the best settings for the second step, we need to put in relation the two steps: if the geometry and the size of the sensitive volume is going to change, 
also the overall geometry and scale of the problem have to change accordingly.

\subsection{Link between the first and second simulations step}

As already specified (Sect. 2.1), particles spectra characterizing the CHARM mixed-field had already been retrieved in the first step simulation (Sect. 2.2). They are now available and will be used as input to perform the second step simulations: the RadFET will be irradiated by a rectangular beam carrying information about the mixedfield in a given position and for a specific target-shielding layout. The beam has the same surface as the RadFET crossing section $\left(1 \mathrm{~mm}^{2}\right)$. In order to give an example, if we want to simulate the dose response of the RadFET, we will irradiate the actual geometry of the MOSFET using a beam that is carrying with itself the spectra of protons, electrons, positrons, muons, ... calculated in the first step simulation, in wanted position target-shielding configuration, available as text files.

The approximation of the radiation field at CHARM as a mono-directional beam, is realistic as the real mixed-field is in good approximation radial. The particles shower originating from the target (and possibly interacting with the shielding, if present) reaches all the rack test positions moving almost along radial directions.

\subsection{The RadFET geometry and materials: their role in the second step}

As it is shown in the Fig. 4, where the yz ( $\mathrm{z}$ is assumed to be the beam direction) and the xy views are shown, the RadFET is mainly composed of a $250 \mu m$ kovar lid, a vacuum region of $250 \mu \mathrm{m}$ and a $500 \mu \mathrm{m}$ Silicon substrate; the crossing plane (xy) surface is $1 \times 1 \mathrm{~mm}^{2}$. A $1.4 \mu \mathrm{m}$ Silicon die is then located on the Silicon substrate: the $\mathrm{SiO}_{2}$ gate oxides we are interested to study are deposited on this thin layer (Fig. 4). The dioxides, 4 in total in our RadFET (named ox1, ox2, ox3, ox4), can be 100 or $400 \mathrm{~nm}$ thick and both the configurations are studied in this paper. For our simulations we took into account just the ox 1 Gate Oxide, having dimensions $300 \mu m \times 50 \mu m$ x 400nm (or $100 \mathrm{~nm}$ ), centered in $\mathrm{x}=0.000 \mathrm{~cm}$ and $\mathrm{y}=-0.0135 \mathrm{~cm}$, with respect to the RadFET center.

The second step approach has been then validated by reproducing the first step configuration (air cube of $20 \mathrm{~cm}$ side) for position 1 in copper target and shielding OFF configuration. We got a deposited dose in the air rack of $1.101 \cdot 10^{-14} \pm 0.7 \% \mathrm{~Gy} /$ pot. This is in perfect agreement with the dose calculated in the $1^{\text {st }}$ step, obtained from a more realistic and complex geometry model of the entire test facility, with a more realistic mixed-field. We are therefore confident that the approximation we introduced to reduce the CPU time, given the microscopic dimensions of our sensitive volume (mixed-field given through a directional beam), is good enough to faithfully reproduce the $1^{\text {st }}$ step.
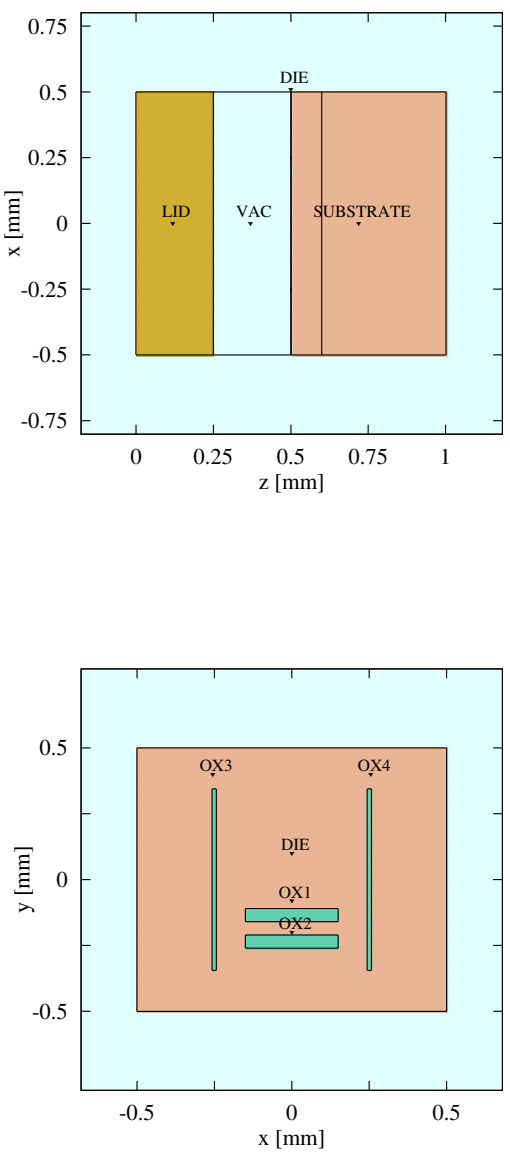

Figure 4: RadFET geometry and materials: FLUKA model.

After this validation of our model, using the $20 \mathrm{~cm}$-side volume of air as scoring volume, we decide to calculate dose in the $6 \cdot 10^{-6} \mathrm{~mm}^{3}$ gate oxide $\left(\mathrm{SiO}_{2}\right)$ of the RadFET, in kovar lid configuration, using the same energy thresholds as before. The simulated dose in this case is $0.895 \cdot 10^{-14}$ $\pm 2.5 \% \mathrm{~Gy} /$ pot, showing a reduction of $19 \%$, if compared to the dose in the air volume.

As clearly visible in Tab. 2, the deposited dose shows

\begin{tabular}{|c|ccc|}
\hline Scoring vol. & 1MeV th. & 1keV th. & \% diff. \\
\hline air cube & $1.101 \pm 0.7 \%$ & $0.840 \pm 0.5 \%$ & $24 \%$ \\
radfet & $0.895 \pm 2.5 \%$ & $0.757 \pm 2.0 \%$ & $15 \%$ \\
\hline
\end{tabular}

Table 2: Dose in $10^{-14} \mathrm{~Gy} /$ pot deposited within the air rack and the RadFET gate oxide in $\mathrm{SiO}_{2}$, using $1 \mathrm{MeV}$ (with NEDEFA defaults) and $1 \mathrm{keV}$ (using PRECISIO defaults) energy thresholds.

a further decrease if we use the PRECISIO physics settings plus $1 \mathrm{keV}$ energy thresholds: in this case the dose is $0.757 \cdot 10^{-14} \pm 2.0 \% \mathrm{~Gy} /$ pot, $15 \%$ lower than the one obtained with the NEWDEFA settings and thresholds at $1 \mathrm{MeV}$ and $31 \%$ smaller than what calculated on the air 
volume, using the same settings as in the $1^{\text {st }}$ step simulation.

This evidently indicates that if we set a more accurate simulation in terms of geometry, materials and energy thresholds, the deposited dose shows a significant decrease with respect to what obtained in the $1^{\text {st }}$ step simulation.

\subsection{Update of the energy thresholds}

As already discussed, the use of the NEWDEFA default in the first step simulation was crucial to reduce the CPU time, given the large geometry of the CHARM test facility. On the other hand, considering that the range of secondary electrons in air is $376 \mathrm{~cm} \mathrm{[12]} \mathrm{and} \mathrm{that} \mathrm{the} \mathrm{characteristic}$ dimension of the air scoring volume we are using is $20 \mathrm{~cm}$, lowering thresholds represents an improvement of the accuracy of our simulations (first row in Tab. 2). It is important to point out, in fact, that in the case of $1 \mathrm{MeV}$ thresholds, we are clearly overestimating the energy deposited, introducing an artifact: all the particles having energies lower than $1 \mathrm{MeV}$ will be treated by the FLUKA Monte Carlo algorithm as depositing their energy on spot, without producing other particles and being transported.

Following the previous considerations, the second step simulations will be then mostly conducted using the PRECISIO default, mainly characterized by particles transport thresholds set at $100 \mathrm{keV}$ (except for neutrons, $10^{-5} \mathrm{eV}$ ) and delta ray production threshold at $100 \mathrm{keV}$. In addition, by using the EMF-CUT and DELTARAY cards, thresholds for transport and production of secondary electrons, positrons and photons and delta ray generated by muons and charged hadrons will be set at $1 \mathrm{MeV}, 100 \mathrm{keV}$, $10 \mathrm{keV}$ and $1 \mathrm{keV}$. The PART-THR card will be instead employed to lower energy transport cut-offs for protons, pions, muons, kaons and $\alpha$ particles to $1 \mathrm{keV}$.

Given the $15 \%$ difference in the dose deposited in the RadFET gate oxide, going from $1 \mathrm{MeV}$ to $1 \mathrm{keV}$ (last row in Tab. 2), it's worth investigating now in which thresholds energy range this main change occurs. The results of this parametric analysis can be found in Tab. 3 .

\begin{tabular}{|c|c|}
\hline Energy threshold & Dose \\
\hline $1 \mathrm{MeV}$ & $0.895 \pm 2.5 \%$ \\
$100 \mathrm{keV}$ & $0.768 \pm 1.5 \%$ \\
$10 \mathrm{keV}$ & $0.787 \pm 1.7 \%$ \\
$1 \mathrm{keV}$ & $0.757 \pm 2.0 \%$ \\
\hline
\end{tabular}

Table 3: Parametric analysis changing the energy thresholds of the simulations (PRECISIO defaults): dose in the $\mathrm{SiO}_{2} \mathrm{GO}$ of the RadFET, in $10^{-14} \mathrm{~Gy} /$ pot.

It is clearly visible that the main change in dose occurs between $1 \mathrm{MeV}$ and $100 \mathrm{keV}$, showing a decrease of $14 \%$. Going down to $10 \mathrm{keV}$ and $1 \mathrm{keV}$ does not considerably change the scenario, the differences are in the range of the statistical uncertainties. This behavior can be explained considering the range of electrons in $\mathrm{SiO}_{2}: 1 \mathrm{MeV}$ electrons have a range of $1.96 \mathrm{~mm}, 100 \mathrm{keV}$ electrons have a range of $66.15 \mu \mathrm{m}$, while $10 \mathrm{keV}$ electrons have a range of $1.23 \mu \mathrm{m}$. By comparing these ranges of secondary electrons with the characteristic lateral dimensions of the gate oxide of the RadFET $(300 \mu \mathrm{m} \times 50 \mu \mathrm{m})$, it is evident that thresholds lower than $100 \mathrm{keV}$ are the most appropriate to simulate and faithfully reproduce the energy deposition in the gate oxide of the RadFET.

In this specific case, since the beam surface has the same size as the crossing surface of the RadFET and the ox 1 sensitive volume is completely irradiated by the beam itself, we have a good statistics using a reasonable number of primaries: we decide to lower the thresholds down to $1 \mathrm{keV}$ to have most precise simulated doses, without loosing big amounts of CPU time.

\subsection{Input spectra cutoffs and oxides thickenss}

After evaluating the dose dependency on materials, scoring volumes sizes and energy thresholds, we decide now to study what is the impact of the oxide thickness and how input spectra cutoffs could affect the final dose.

We simulate RadFET having $100 \mathrm{~nm}$ and $400 \mathrm{~nm}$ thick gate oxides. We reproduce, on the other hand, input spectra characterizing the mixed-field, through a dedicated and independent first step simulation, by cutting them at $1 \mathrm{MeV}$ and $100 \mathrm{keV}$, to see how lower energy particles of the spectra could affect the final deposited dose. Going down to energies lower than $100 \mathrm{keV}$ is counterproductive: the lower the thresholds, the lower the ranges and that particles can stopped by the kovar lid of the RadFET. As clearly visible from Tab. 4, the oxide thickness does not have a large impact on the simulated deposited dose. On the other hand, using spectra cut at $1 \mathrm{MeV}$ or $100 \mathrm{keV}$ makes a difference that is of the order of $10 \%$.

\begin{tabular}{|l|lll|}
\hline Thickness & 1MeV input & 100keV input & \% diff. \\
\hline $400 \mathrm{~nm}$ & $0.757 \pm 2.0 \%$ & $0.820 \pm 4.3 \%$ & $8 \%$ \\
$100 \mathrm{~nm}$ & $0.719 \pm 3.8 \%$ & $0.816 \pm 2.4 \%$ & $12 \%$ \\
\hline
\end{tabular}

Table 4: Effect of Gate Oxide thickness and input spectra thresholds on the RadFET dose response: simulated dose in $10^{-14} \mathrm{~Gy} /$ pot (PRECISIO defaults set).

\section{Second step simulations: the cp_0000 target-shielding configuration}

The analyses conducted in Sect. 3 led us to identify the following simulation settings as the most suitable for our second step simulations:

- 400nm thick oxide;

- PRECISIO default in FLUKA;

- Energy thresholds at $1 \mathrm{keV}$;

- Input spectra cutoffs at $100 \mathrm{keV}$.

This is the starting point to test the second step algorithm on other relevant positions inside the test area, concerning the cp_0000 target-shielding configuration. 


\subsection{Doses simulated and measured in different positions at CHARM}

We set the RadFET to positions 3, 5, 7, 10 and 13, by using as input the spectra of the $1^{\text {st }}$ step simulations, calculated for the new locations of interest, as described in Sect. 2.2.

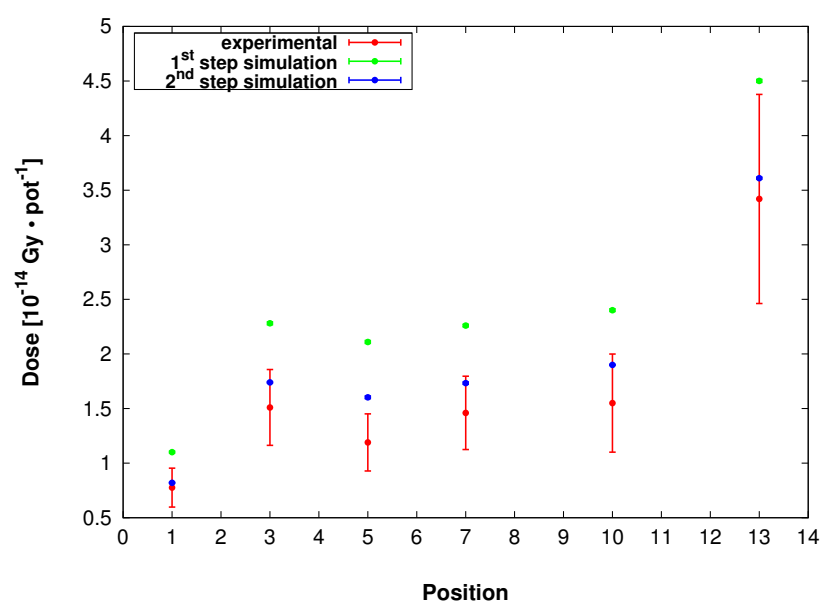

Figure 5: Experimental and simulated doses- $1^{\text {st }}$ and $2^{\text {nd }}$ step simulations- at different test positions, cp_0000 configuration.

Fig. 1 shows how the new considered locations are differently exposed to the mixed-field, mostly because of geometric reasons: we expect the position 13 to be actually the most solicited one, since directly exposed to the flux and almost in the beam direction. In addition, we want to compare simulated doses to experimental data (Sect. 2.3), to show the improvements we got by performing a more accurate second step simulation.

Tab. 5 clearly shows how the difference between experimental data and simulated values is now lower if compared to what obtained in Sect. 2.3, Tab. 2. The mismatch measured-simulated is $5 \div 25 \%$ - compatible with experimental uncertainties instead of $25 \div 43 \%$, showing a substantial reduction $(20 \div 25 \%)$ of the simulated dose (Tab. 6), in the direction of experimental data.

It is then evident that the new simulation allowed us to obtain most accurate simulated values, in better agreement with the measured doses. This is also clear from Fig. 7, where experimental, $1^{\text {st }}$ and $2^{\text {nd }}$ step doses are plotted: the second step simulation has represented an important improvement in simulating the radiation field at CHARM in the copper target and shielding OFF configuration.

\begin{tabular}{|l|lll|}
\hline Position & Exp. dose & $2^{\text {nd }}$ step dose & \% diff. \\
\hline $\mathbf{1}$ & $0.776 \pm 23.0 \%$ & $0.820 \pm 0.6 \%$ & $5 \%$ \\
$\mathbf{3}$ & $1.510 \pm 23.0 \%$ & $1.740 \pm 0.4 \%$ & $13 \%$ \\
$\mathbf{5}$ & $1.190 \pm 22.0 \%$ & $1.603 \pm 0.8 \%$ & $25 \%$ \\
$\mathbf{7}$ & $1.460 \pm 23.0 \%$ & $1.733 \pm 0.6 \%$ & $15 \%$ \\
$\mathbf{1 0}$ & $1.550 \pm 29.0 \%$ & $1.900 \pm 0.2 \%$ & $18 \%$ \\
$\mathbf{1 3}$ & $3.420 \pm 28.0 \%$ & $3.610 \pm 0.3 \%$ & $5 \%$ \\
\hline
\end{tabular}

Table 5: Experimental and $2^{\text {nd }}$ step simulated doses in $10^{-14} \mathrm{~Gy} / \mathrm{pot}$, in different positions at CHARM.

\begin{tabular}{|l|lll|}
\hline Position & $1^{\text {st }}$ step dose & $2^{\text {nd }}$ step dose & \% diff. \\
\hline $\mathbf{1}$ & $1.101 \pm 0.8 \%$ & $0.820 \pm 0.6 \%$ & $25 \%$ \\
$\mathbf{3}$ & $2.281 \pm 0.6 \%$ & $1.740 \pm 0.4 \%$ & $24 \%$ \\
$\mathbf{5}$ & $2.110 \pm 0.6 \%$ & $1.603 \pm 0.8 \%$ & $24 \%$ \\
$\mathbf{7}$ & $2.260 \pm 0.5 \%$ & $1.733 \pm 0.6 \%$ & $23 \%$ \\
$\mathbf{1 0}$ & $2.401 \pm 0.5 \%$ & $1.900 \pm 0.2 \%$ & $21 \%$ \\
$\mathbf{1 3}$ & $4.510 \pm 0.3 \%$ & $3.610 \pm 0.3 \%$ & $20 \%$ \\
\hline
\end{tabular}

Table 6: $1^{\text {st }}$ and $2^{\text {nd }}$ simulated doses in $10^{-14} \mathrm{~Gy} /$ pot, in different positions at CHARM.

\subsection{Contribution of each mixed-field's particles to the total dose}

After validating the $2^{\text {nd }}$ step simulation settings for the cp_0000 target-shielding configuration for different positions inside the test facility, the simulations results of the single particles (of the mixed-field) contribution to the total dose will be presented. The dose coming from all the particles of the mixed-field is going to be tabulated for the position 1,3,10 and 13; particular attention will be given to the weight of each particle over the total dose.

As showed in Fig. 6, downstream positions $(10 \div 13)$ are the most exposed to the particle shower developing after the beam-target interaction. Speaking in terms of doses deposited by the different particles of the mixed-field, the energies deposited by charged hadrons, electrons and positrons, muons increase going from location 1 to 13 (Fig. 6). On the other hand, doses coming from neutrons and photons are almost constant. The explanation has to be found in the topography of our radiation field: fluences of charged hardons, electrons and positrons an muons are in fact characterized by a forward directionality, while, on the contrary, photons and neutrons fluences are more isotropic.

Considering the relative contribution of each particle of the field over the total dose (Fig. 7), it's interesting to note how, going from lateral to downstream positions, from 1 to 13:

- Charged Hadrons (protons, pions, kaons) contribution rises from $34 \%$ to $48 \%$;

- Electrons and positrons contribution rises from $30 \%$ to $40 \%$;

- Muons doses are stable around the 3-4\%, representing an almost negligible portion of the total;

- Photons doses decrease from $24 \%$ to $7 \%$ in terms of contribution to the total;

- Neutrons contribution goes down from $8.5 \%$ to $2.5 \%$ in terms of contribution to the total. 


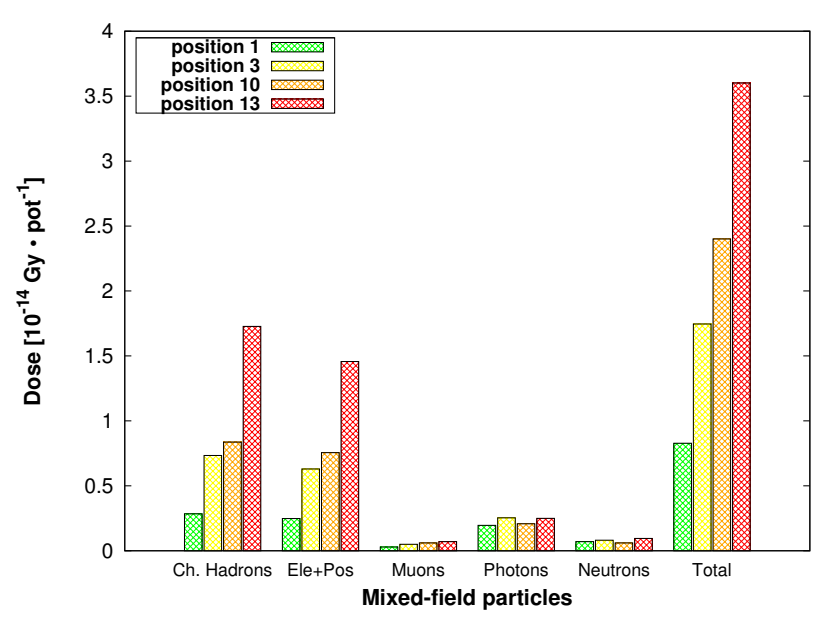

Figure 6: Particles contribution to the total dose: dose values.

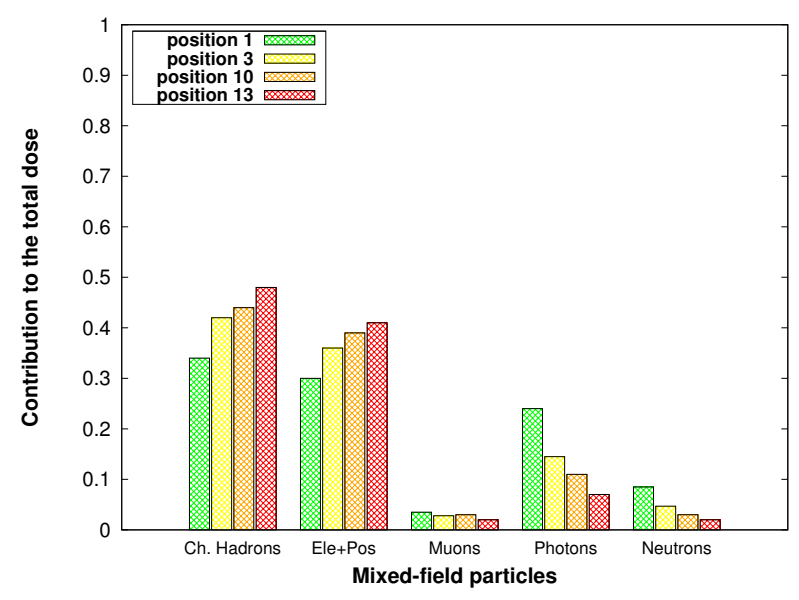

Figure 7: Particles contribution to the total dose: relative contribution.

As already said, this is a specular way to see how going from lateral to upstream positions, the contributions of the primary particles of the mixed-field to the total dose change and in particular charged hadrons, electrons and positrons become dominant, while photons and neutrons weights loose relevance.

\section{5 cp_CIIC target-shielding configuration: the thermal neutrons issue}

In this paragraph we will use the same simulation settings employed in the frame of the cp_0000 target-shielding configuration, for the cp_CIIC layout.

As for the previous case, we will irradiate the RadFET with a beam carrying information about the different spectra of the mixed-field, starting from position 1 .

\subsection{Doses simulated and measured in position 1}

We want to compare the two step simulations results. As visible in Tab. 7, where doses for position 1 and cp_CIIC are reported, the mismatch between first and second step doses is now $52 \%$. This could mean that probably something is missing in our model.

Given the unexpected discrepancy, we decide to evaluate

\begin{tabular}{|ll|l|}
\hline $\mathbf{2}^{\text {nd }}$ step & $\mathbf{1}^{\text {st }}$ step & \%diff. \\
\hline $0.0327 \pm 0.6 \%$ & $0.0678 \pm 0.8 \%$ & $52 \%$ \\
\hline
\end{tabular}

Table 7: Experimental, $2^{\text {nd }}$ and $1^{\text {st }}$ step simulated doses in $10^{-14} \mathrm{~Gy} /$ pot, in position 1, cp_CIIC configuration.

the single contributions to the total dose, due to all the particles populating the CHARM mixed-field, in cp_CIIC configuration, in order to investigate this issue. In this regard, Fig. 8 shows a comparison between the two step in the air cube of $20 \mathrm{~cm}$ side (taken as a reference) and the RadFET gate oxide, using the same settings as in Sect. 3.1. It's worth noticing that the doses deposited by charged hadrons, muons and electrons+positrons+photons are perfectly comparable in both cases (air volume and RadFET). What really changes is the dose contribution due to neutrons: the dose simulated in the large air volume is a factor 5.3 higher than what scored in the RadFET.

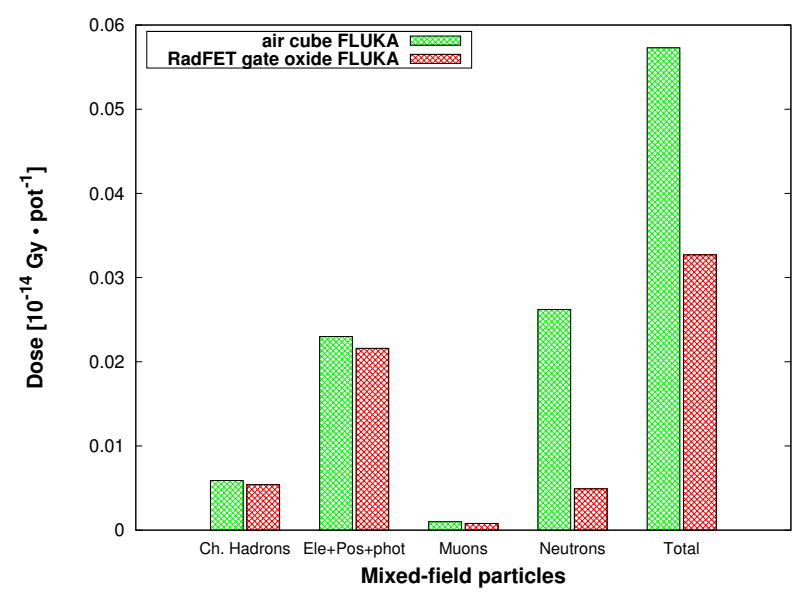

Figure 8: Particles contribution to the total dose: dose values.

\subsection{Splitting neutrons spectrum: contribution at different energies}

For the reasons exposed in the previous paragraph, we decide to split the spectrum of neutrons in different energy ranges, to further investigate where this mismatch could be originated. From Tab. 8 it's clear that the discrepancy we got is mainly due to thermal neutrons, which are depositing in the RadFET gate oxide a dose that is a factor 50 lower than expected (dose simulated in the air volume and measurements). This is, anyway, something in line with expectations: thermal neutrons undergo np nuclear interactions with the nuclei of Nitrogen atoms present in air and these interactions give birth to protons at $300 \div 400$ $\mathrm{keV}$ that are actually depositing energy in the air cube volume. 


\begin{tabular}{|l|ll|l|}
\hline Energy range & Air Vol. dose & RadFET dose & ratio \\
\hline $\mathrm{E}_{n} \leqslant 0.1 \mathrm{eV}$ & $0.0151 \pm 0.5 \%$ & $0.0003 \pm 8.5 \%$ & 50.0 \\
$0.1 \mathrm{eV}<\mathrm{E}_{n}<20 \mathrm{MeV}$ & $0.0087 \pm 2.5 \%$ & $0.0029 \pm 4.9 \%$ & 3.0 \\
$\mathrm{E}_{n} \geqslant 20 \mathrm{MeV}$ & $0.0024 \pm 5.5 \%$ & $0.0017 \pm 5.5 \%$ & 1.4 \\
Full spectrum & $0.0262 \pm 1.5 \%$ & $0.0049 \pm 4.0 \%$ & 5.4 \\
\hline
\end{tabular}

Table 8: Contributions to the total dose deposited in the air cube and the RadFET GO, in $10^{-14} \mathrm{~Gy} /$ pot, in position 1 , due to neutrons at different energies.

On the other hand, this is evidently not the case if we consider the dose simulated in the RadFET gate oxide: before reaching the oxide, thermal neutrons have interacted with the Kovar (Ni-Co) lid (possibly developing a further particles shower) and finally deposited energy in $\mathrm{SiO}_{2}$, without interacting with any air molecule.

This is clearly only partially explaining the discrepancy we found and further investigations are already ongoing, laying the basis for the next step of research. In the next future, in fact, we want to build a most accurate model of the RadFET in terms of materials, thinking, for instance, that the doping could be a potential source of this discrepancy. In parallel, new test runs at CHARM will give us more information and most reliable measurements about the dose absorbed by RadFETs in the cp_CIIC configuration. Also a test campaign to measure the RadFET sensitivity to low energy neutrons will take place in the early 2017 .

\section{Summary and conclusions}

By implementing the real geometry of the RadFET in FLUKA, using most accurate physics settings and input spectra from the first simulation step, we are able to simulate the RadFET dose response in the CHARM mixedfield- copper target and shielding OFF configuration- with a high accuracy. Through the second simulations step, we got a reduction of the mismatch between measured and simulated doses of $20 \div 25 \%$. Equivalently, simulated doses are definitely closer to the experimental data $(5 \div 25 \%$ difference between the two), than what had already been done with the previous calculations, which showed a difference of $25 \div 45 \%$ between simulated and measured values.

On the other hand, the same simulation settings do not seem to be entirely applicable when simulating the dose response of the RadFET under the mixed-field generated in copper target and concrete-iron-iron-concrete shielding configuration. From the simulations we conducted, this is apparently due to the thermal neutrons component of the radiation field at CHARM (dominant in the shielded case and evidently negligible in th cp_0000 configuration) and requires further investigation both from the experimental and simulation point of view. In this regards, this is the starting point for the next future research, aiming at finding unambiguous simulation settings to be used under different target-shielding configurations at CHARM and confirmed by experimental measurements.

\section{References}

[1] A. Ferrari et al., "FLUKA: a multi-particle transport code", CERN-2005-10 (2005), INFN/TC05/11, SLACR-773

[2] T.T. Böhlen et al. "The FLUKA Code: Developments and Challenges for High Energy and Medical Applications", Nuclear Data Sheets 120, 211-214 (2014)

[3] P. Beck et al., "Investigation on Photon Energy Response of RadFET Using Monte Carlo Simulations", IEEE Transactions on nuclear science, Vol. 54, no. 4, pp. 1151-1157, August 2007.

[4] M. Wind et al., "Investigation of the Energy Response of RadFET for High Energy Photons, Electrons, Protons, and Neutrons", IEEE Transactions on nuclear science, Vol. 56, no. 6, pp. 3387-3392, December 2009.

[5] G. Spiezia et al., "The LHC Accelerator Radiation Monitoring System-RadMon", 2011.

[6] J. Mekki et al., "Mixed Particle Influence on RadFET Responses Using Co-60 Calibration", IEEE Transactions on nuclear science, Vol. 60, no. 4, pp. 2435-2443, August 2013.

[7] G. Spiezia et al., "A New RadMon Version for the LHC and its injection lines", IEEE Trans. Nucl. Sci, vol. 61, no. 6, pp. 3424-3431, 2014.

[8] J. Mekki et al., "A Mixed Field Facility At CERN for Radiation Test: CHARM", RADiation and its Effects on Components and Systems (RADECS), 14-18 September 2015.

[9] A. Holmes-Sieldle and L. Adams, "Handbook of Radiation Effects", Second Edition, Oxford University Press, 2002.

[10] S. Bonaldo, "CHARM Una nuova facility del CERN per test di elettronica con spettri misti di radiazione ionizzante", 2016.

[11] F. Ravotti et al., "Response of RadFET Dosimeters to High Fluences of Fast Neutrons", IEEE Transactions on nuclear science, Vol. 52, no. 4, pp. 959-965, August 2005.

[12] Martin J. Berger et al., "ESTAR, PSTAR, and ASTAR: Computer Programs for Calculating Stopping-Power and Range Tables for Electrons, Protons, and Helium Ions (version 1.21)", Citation: http://physics.nist.gov/Star 